\title{
Software Defined Radio for Underwater Applications using FPGA
}

\author{
${ }^{1}$ Ch.Sailaja, ${ }^{2}$ D.Rajaramesh, ${ }^{3}$ M.Srinivasa Rao \\ ${ }^{I}$ M.Tech Student, IEEE Member, M.V.G.R.College of Engineering, Vizianagaram \\ ${ }^{2}$ Assistant Professor, M.V.G.R.College of Engineering,Vizianagaram \\ ${ }^{3}$ Scientist ' $F$ ', NSTL, Visakhapatnam
}

\begin{abstract}
This paper describes an Underwater Acoustic Communication system for establishing a two-way communication between monitoring station and the underwater device. In order to obtain high data rates with utilization of an effective bandwidth, Orthogonal Frequency Division Multiplexing Technique (OFDM) has been chosen. With the proposed OFDM parameters, we have done an offline analysis of the data and had even verified the results using an existing modem.
\end{abstract}

Keywords: OFDM; FFT; FPGA; QPSK; SDR; ISI.

\section{Introduction}

Recent advances in underwater acoustic communication led to the research in the implementation of a Software-defined Radio (SDR). Due to the various applications and advantages, underwater acoustic communication has gained immense research attention from the past few decades. The tremendous desire to cope up with the hardware complexity and to make the entire system a cost-effective one, SDR has evolved where the entire process of the system is implemented in software itself.

Ocean environment has limited bandwidth due to its time-varying nature of the channel. Acoustic waves propagate with very less attenuation. Inorder to transmit large data in the same amount of time with larger bandwidth, Orthogonal Frequency Division Multiplexing (OFDM) has been chosen.

In an OFDM based acoustic communication system, the entire bandwidth is divided into a large number of narrow sub-channels thus making each of these channels into flat-fading.

OFDM is robust to InterSymbol Interference (ISI), Intercarrier Interference (ICI), and Doppler Effect as far as orthogonality among each subcarrier is preserved. Inorder to implement an SDR, some modules are implemented in Digital Signal Processor (DSP) and some in a Field Programmable Gate Array (FPGA).

The reconfigurable nature, short time to market has made system designers to choose an FPGA.

\section{Software Defined Radio (SDR)}

The tremendous need and demand for wireless communication in underwater environment has led to the evolution of Software defined Radio (SDR). Integrating large number of components onto a single circuit is known as a Very Large Scale Integrated Circuit (VLSI) that resulted in very low cost, size, power consumption, high flexibility and reconfigurable logic.

The inherent capability of SDR to adapt different logics onto a single system with less hardware complexity and the tremendous flexibility to change the logic in software at the cost of very less logic resources and less time-to-market has led the recent research in underwater acoustic communication to migrate from the previous methodologies to this technology.

Depending on the wireless communication standard being supported, the processing demands vary significantly, resulting in software-only systems or software-plus-hardware based systems. These systems will be implemented in a combination of digital signal processors and FPGA based dedicated logic architectures.

\section{Underwater Acoustic Communication}

Communication in underwater has gained immense interest from the last few decades due to its various applications. However, the underwater channel is bandlimited and sparse in nature. Therefore communication in underwater is a very tedious task. Several researches have been done to achieve higher data rates with very less bit error rate. researches.

Orthogonal Frequency Division Multiplexing (OFDM) technique has evolved as a result of these

Underwater acoustic communication has many applications however this project is aimed for the telemetry application. This paper is in support with my previous paper "Implementation of Fast Fourier Transform for Underwater Applications using FPGA". 
Underwater channel has lot of problems like the multipath propagation, Doppler Effect, high ISI, low SNR, ambient noise and external interferences. Inorder to overcome these channel adverse effects, an effective multicarrier modulation technique has been chosen that avoids ISI and other problems.

In an underwater acoustic communication system the major blocks include the FFT/IFFT, channel estimation and channel equalizer.

The modulation technique that has been selected for the subcarriers in OFDM is Quadrature Phase Shift Keying (QPSK) technique. OFDM has the distinct advantage that it converts the frequency-selective fading into flat-fading channels. Therefore, high spectral utilization is achieved.

\section{Adaptive Filters}

The system that carries out and undergoes the process of "adaptation" i.e. adjusting itself to meet the target depending on its state and environment and finally reshaping the frequency components of the input to generate the output is often termed as adaptive filters. These filters play a vital role in the underwater acoustic communication where the channel response is time-varying in nature.

These are digital filters and depending on the convergence time it takes, several algorithms and filter structures has been developed. A good filter will always give a good estimate of the given desired signal. In our application, we require the length of the adaptive filter to be a hundreds or even a few thousands of taps.

The conventional Least Mean Squares (LMS) algorithm is computationally expensive to implement. Therefore, in such cases, we chose block LMS that significantly reduces the complexity. In this block processing, a block of input samples and desired samples are collected and then processed together to obtain the output samples.

\section{OFDM}

As illustrated in my previous paper "Implementation of Fast Fourier Transform for Underwater Applications using FPGA", the basic steps that are involved in the implementation of OFDM is given below. The block diagram of the OFDM is as shown in fig 1.

Once the time synchronization is achieved, the received waveform is divided into symbols and is applied to OFDM demodulator with adaptive equalizer to estimate symbols transmitted.

This decoded message can be suitable for command, control and monitoring the activities of underwater data acquisition devices.

\section{A. Design}

OFDM based Underwater Acoustic Communication is implemented efficiently on DSP and FPGA. The efficiency of such a communication system is predominantly dependent on the architecture of FFT we choose. Here Radix-2 Lite Burst I/O architecture was chosen for the implementation of both FFT and IFFT. It uses a time-multiplexed approach to butterfly for even smaller core, at the cost of longer transform time.

On the transmitter side we initially map the message bits into symbols based on the type of modulation we choose for the subcarriers. Once they are mapped according to the constellation, the serial stream of bits is converted into parallel so as to feed to the IFFT block.

Here the frequency domain data is transformed into time-domain and finally a parallel to serial block is used so as to make the data to be transmitted over the channel. In this process of transmitting data, synch pulses are added along with the data so as to make it feasible for the receiver to have a better synchronization. These synch pulses along with the other pulses i.e., header synch, Doppler synch are all chirp signals.

On the receiver side we have channel estimation and equalizer, synchronization and FFT blocks which play a vital role in any communication system. Channel estimation is important as without identifying the characteristics of the channel it would be an hectic job to perform the right analysis of the transmitted data. An equalizer is needed so as to adjust itself according to the desired response.

To perform this complete OFDM based acoustic communication we have transmitted a 60 bit message stream and implemented on the DSK. The proposed OFDM parameters as illustrated in my previous paper "Implementation of Fast Fourier Transform for Underwater Applications using FPGA" are considered the same. 


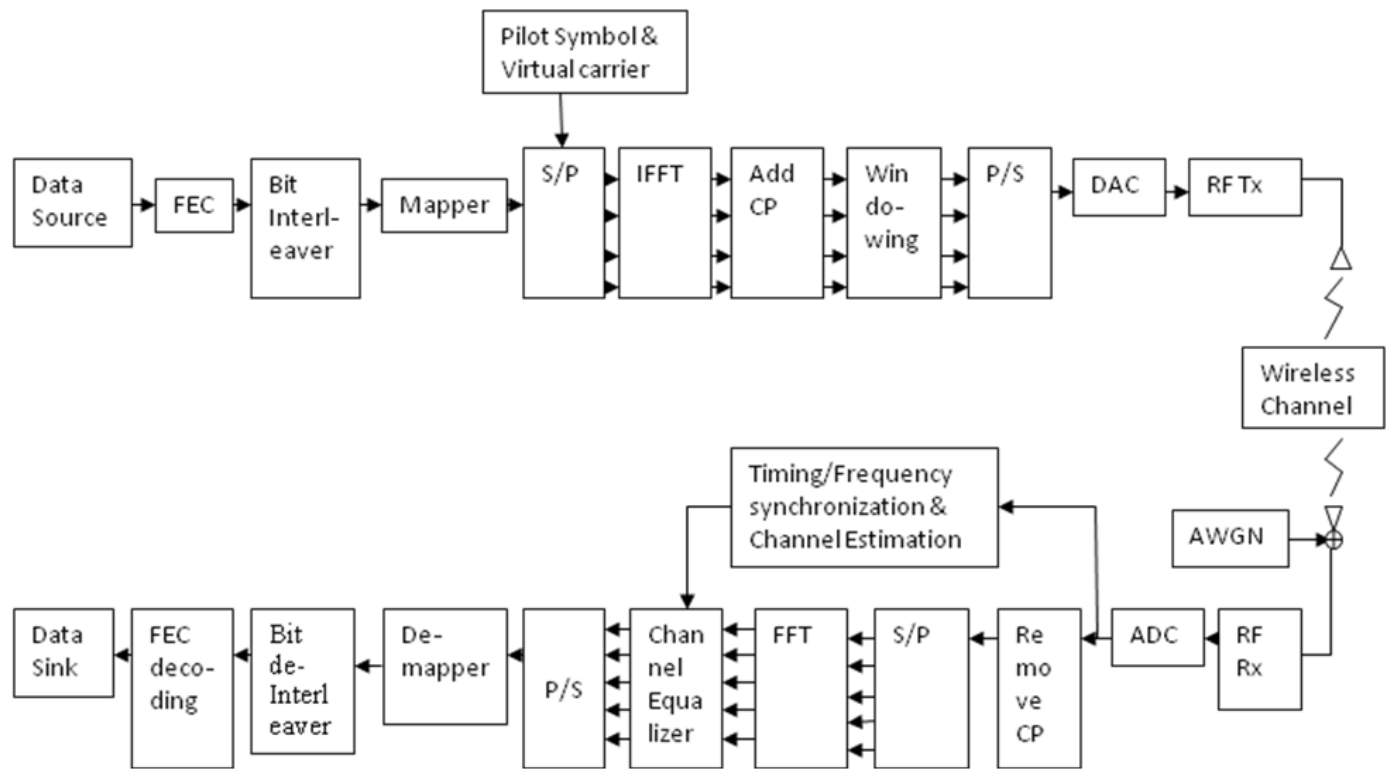

Fig 1: Underwater OFDM Communication system

Table 1: Proposed OFDM Parameters

\begin{tabular}{|l|l|}
\hline Sampling Frequency & 48000 \\
\hline FFT and IFFT length & 1024 \\
\hline Guard Time / Cyclic Prefix & $32 \mathrm{bits}$ \\
\hline Sub-carrier Modulation & DQPSK \\
\hline $\begin{array}{l}\text { Symbol Duration (without } \\
\text { cyclic prefix) }\end{array}$ & $32 \mathrm{msec}$ \\
\hline $\begin{array}{l}\text { Symbol Duration (with } \\
\text { cyclic prefix) }\end{array}$ & $34 \mathrm{msec}$ \\
\hline Number of Carriers used & 43 \\
\hline Carrier Spacing & $47 \mathrm{~Hz}$ \\
\hline Frequency Band of usage & $15984.375 \quad$ to 18000 \\
\hline
\end{tabular}

\section{Experimental Results}

In the experimental setup, we have a Power amplifier, transducers, pre-amplifier and a data acquisition system. The data acquisition system consists of an A/D converter, FPGA and a memory to store the data. The signal from the DSK6713 is fed to the transducers through a power amplifier and at the receiving side, the low electric signal is pre-amplified before passing it to the data acquisition system.

\section{Experimental Setup of OFDM Receiver}

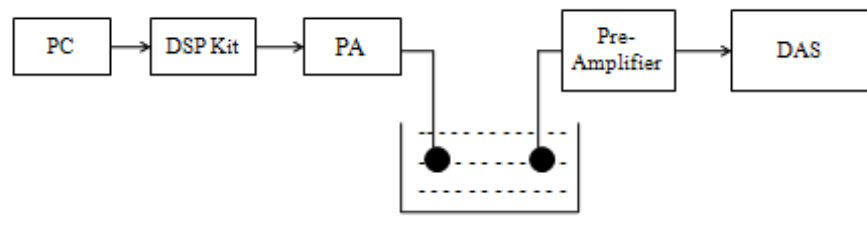

PA: Power Amplifier

Data Acquisition System (DAS)

DSK Kit: TMS320C6713

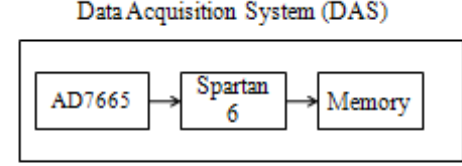

Fig2: Experimental Setup of OFDM Receiver. 


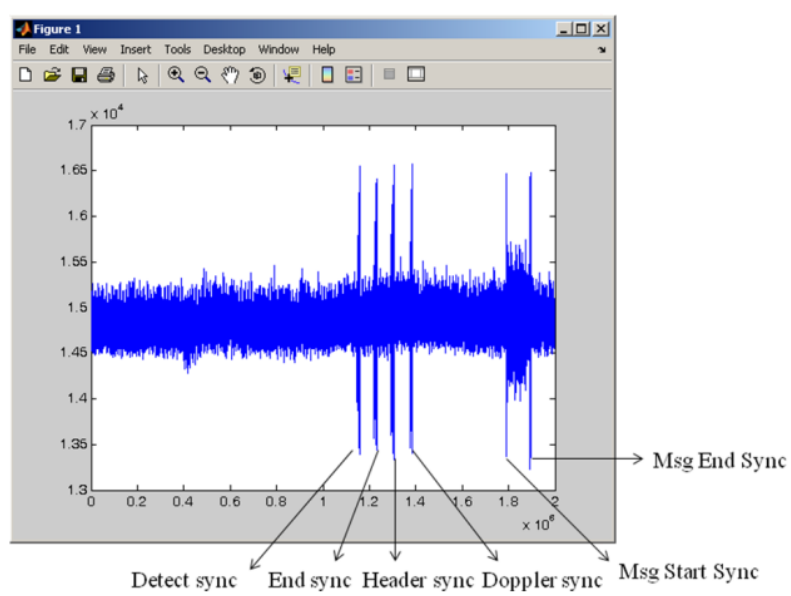

Fig 3: OFDM Transmitted signal using MODEM.

The transmitted message data is captured in a CRO and is shown in the below fig.4.

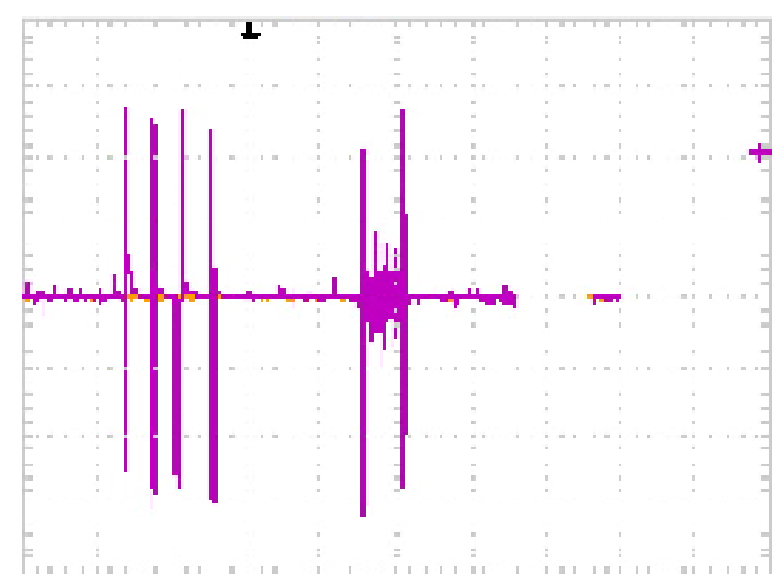

Fig 4: OFDM Transmitted Signal

\section{Acknowledgment}

Our sincere thanks to Sri.C.D.Malleswar, Scientist-G, Director, Naval Science and Technological Laboratory for his encouragement and support in bringing out this paper. Thanks are also due to Sri S.Raja Scientist-G of NSTL for his encouragement in bringing this paper. The authors sincerely acknowledge the contributions made by other team members.

\section{References}

[1] Walter Tuttlebee, "Software Defined Radio: Baseband Technologies for 3G Handsets and Basestations", John Wiley \& Sons Ltd2004 Edition.

[2] FFT v8.0 IP core from www.xilinx.com.

[3] Shengli Zhou, Zhaohui Wang, "OFDM for Underwater Acoustic Communications”, Wiley Edition.

[4] Yong Soo Cho, Jaekwon Kim,Won Young Yang, Chung G. Kang "MIMO-OFDM Wireless communications with MATLAB" John Wiley \& Sons (Asia) Pte Ltd-2010 Edition.

[5] Rulph Chassaing, "Digital Signal Processing and Applications with the C6713 and C6416 DSK", Wiley Edition.

[6] U.Meyer Baese, "Digital Signal Processing with Field Programmable Gate Arrays", Springer $3{ }^{\text {rd }}$ Edition.

[7] TLV320AIC23B Data Manual of Texas Instruments, Febuary 2004.

[8] Shengli Zhou,Zhaohui Wang, "OFDM for Underwater Acoustic Communications", Wiley Ed.,2014. 